\title{
Gate-tunable supercurrent and multiple Andreev reflections in a superconductor-topological insulator nanoribbon-superconductor hybrid device
}

\author{
Luis A. Jauregui, 1, a) Morteza Kayyalha, 1,b) Aleksandr Kazakov, ${ }^{2}$ \\ Ireneusz Miotkowski, ${ }^{2}$ Leonid P. Rokhinson, ${ }^{2,1}$ and Yong P. Chen ${ }^{2,1,3,}$ c) \\ 1) School of Electrical and Computer Engineering and Birck Nanotechnology Center, \\ Purdue University, West Lafayette, IN 4790\%, USA \\ ${ }^{2)}$ Department of Physics and Astronomy, Purdue University, West Lafayette, IN 47907 , \\ USA \\ 3) Purdue Quantum Center, Purdue University, West Lafayette, IN 47907, \\ $U S A$
}

(Dated: 11 October 2017)

\begin{abstract}
We report on the observation of gate-tunable proximity-induced superconductivity and multiple Andreev reflections (MAR) in a bulk-insulating BiSbTeSe $\mathrm{B}_{2}$ topological insulator nanoribbon (TINR) Josephson junction (JJ) with superconducting $\mathrm{Nb}$ contacts. We observe a gate-tunable critical current $\left(I_{C}\right)$ for gate voltages $\left(V_{g}\right)$ above the charge neutrality point $\left(V_{C N P}\right)$, with $I_{C}$ as large as $430 \mathrm{nA}$. We also observe MAR peaks in the differential conductance $(d I / d V)$ versus DC voltage $\left(V_{d c}\right)$ across the junction corresponding to subharmonic peaks (at $V_{d c}=V_{n}=2 \Delta_{N b} / e n$, where $\Delta_{N b}$ is the superconducting gap of the $\mathrm{Nb}$ contacts and $n$ is the sub-harmonic order). The sub-harmonic order, $n$, exhibits a $V_{g}$-dependence and reaches $n=13$ for $V_{g}=40 \mathrm{~V}$, indicating the high transparency of the $\mathrm{Nb}$ contacts to TINR. Our observations pave the way toward exploring the possibilities of using TINR in topologically protected devices that may host exotic physics such as Majorana fermions.
\end{abstract}

Three-dimensional topological insulators (TI's) are a new class of quantum matter with an insulating bulk and conducting surface states, topologically protected against time-reversalinvariant perturbations (scattering by non-magnetic impurities such as crystalline defects and surface roughness) 12 . Topological superconductors (TSC's) are another important class of quantum matter and are analogous to TI's, where the superconducting gap and Majorana fermions of TSC's replace the bulk bandgap and Dirac fermion surface states of the TI, respectively 2 . Controlling the Majorana modes is considered one of the important approaches for developing topologically protected quantum computers. Three-dimensional (3D) TIs in proximity to s-wave superconductors have been proposed as one of the promising platforms to realize topological superconductivity and Majorana fermions 3 . In this context, it has been pointed out that TI nanowires (TINWs) possess various appealing features for such studies 48. However, the first important step is to understand how TI nanowires, including nanoribbons (TINR's), behave in contact with superconducting leads.

Superconductor normal superconductor (SNS) Josephson junctions(JJs), with topological insulators as the normal material have been experimentally realized on 3D-TI's 22 . However, TI materials used in many of the previous experiments have notable bulk conduction, making it challenging to distinguish from the contribution of the topological surface states. In this letter, we study S-TINR-S Josephson junctions, where $\mathrm{S}=$ Niobium $(\mathrm{Nb})$ and the TINR's are mechanically exfoliated from bulk BiSbTeSe 2 (BSTS) TI crystals. Our BSTS is among the most bulk-insulating TI's with surface states dominated conduction, and chemical potential located close to the surface state Dirac point in the bulk bandgap $23 \mid 24$. Therefore, our study enables us to investigate the proximity effects and induced superconductivity in such "intrinsic" (bulk-insulating) and gate-tunable TINR's with both electron $(\mathrm{n})$ and hole $(\mathrm{p})$ dominated surface transport. Moreover, we are able to investigate the

\footnotetext{
a) These authors contributed equally; Current address: Department of Physics, Harvard University, Cambridge, MA 02138, USA

b) These authors contributed equally

c) Electronic mail: yongchen@purdue.edu
} 

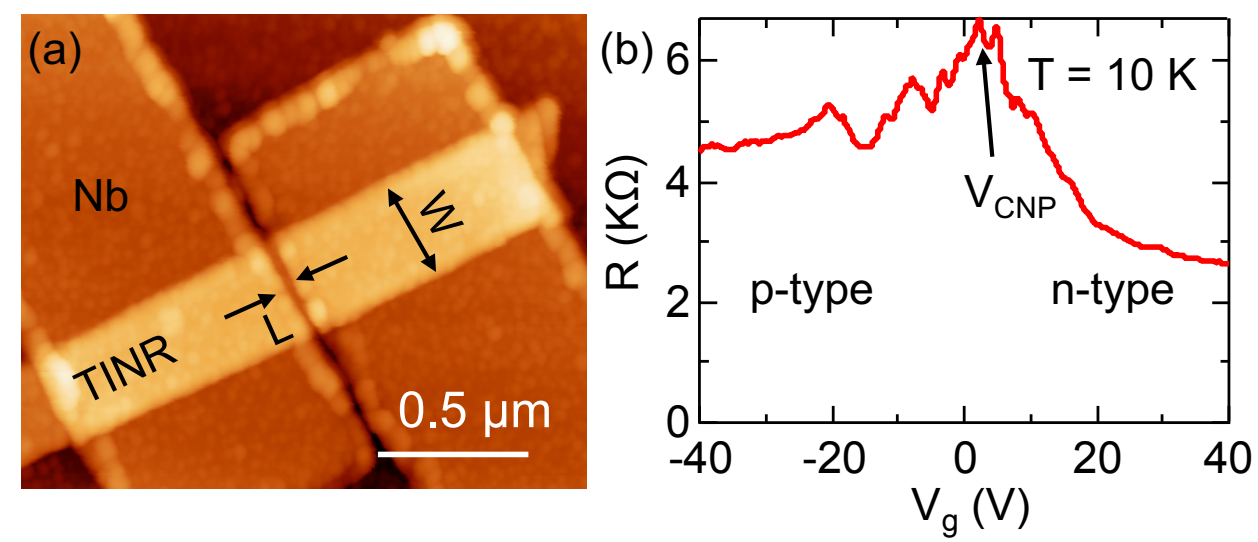

FIG. 1. (a) Atomic force microscope (AFM) image of a 250-nm wide and 20-nm thick TINR multiterminal device with $\mathrm{Nb}$ electrodes (electrode separation $\mathrm{L} \sim 60 \mathrm{~nm}$ ). (b) Two-terminal resistance $(R)$ vs. the back-gate voltage $\left(V_{g}\right)$, measured at $T=10 \mathrm{~K}$, above the critical temperature $\left(T_{C}^{N b}\right)$ of the $\mathrm{Nb}$ electrodes.

transparency of our superconducting contacts to TINR both in n- and p- dominated transport regimes through the observation of multiple Andreev reflections (MAR).

High-quality single crystals of BiSbTeSe $_{2}$ (BSTS) were grown by the Bridgman technique as described elsewhere ${ }^{2324}$. Devices fabricated on the exfoliated flakes from these crystals exhibit surface dominated conduction with ambipolar field effect, half-integer quantum hall effect, and $\pi$-Berry phase ${ }^{2324}$. We obtain BSTS nanoribbons using a standard mechanical exfoliation technique and transferred them onto a 500- $\mu \mathrm{m}$ thick highly doped Si substrate (used as the back gate) covered with 300-nm $\mathrm{SiO}_{2}$ on top. We locate BSTS nanoribbons, which are randomly dispersed on the substrate, by an optical microscope. An atomic force microscope (AFM) image of a representative JJ is shown in Fig 1a. Multiple electrodes, with electrode separation $L<100 \mathrm{~nm}$ between the adjacent electrodes, are defined by e-beam lithography for each TINR. We then deposit 30-nm thick Nb contacts by a DC sputtering system. A short ( $\sim 5 \mathrm{sec})$ in situ Ar ion milling prior to the metal deposition is used to remove any residues left from the lithography step and native oxides on the TINR surface. Our results presented here are taken from a TINR sample with a thickness of $\sim 20 \mathrm{~nm}$, width of $\sim 250 \mathrm{~nm}$, and electrode separation of $\sim 60 \mathrm{~nm}$.

Fig. $1 \mathrm{~b}$ depicts $R$ vs. the back-gate voltage $\left(V_{g}\right)$ at $T=10 \mathrm{~K}$ (above the critical temperature of our deposited superconductor, $T_{C}^{N b} \sim 6.5 \mathrm{~K}$ ). The charge neutrality-point voltage $\left(V_{C N P}\right)$ is $\sim 4 \mathrm{~V}$ for this device. The electron- and hole-dominated regimes can be easily observed in Fig. $1 \mathrm{~b}$ as we tune $V_{g}$ away from the $V_{C N P}$. Using BCS theory, we estimate the $T=0 \mathrm{~K}$ superconducting gap as $\Delta_{N b}=1.76 k_{B} T_{C}^{N b} \sim 975 \mu \mathrm{eV}$.

When the sample is cooled down below $T_{C}^{N b}$, the electronic transport in the junction is strongly affected by the superconducting proximity effect. The evidences of this effect manifest themselves as the flow of a supercurrent in the junction and the appearance of multiple Andreev reflections (MAR) ${ }^{25126}$. Fig. 2a shows the colormap of the differential resistance $(d V / d I)$ vs. $V_{g}$ and $I_{d c}$ at $T=30 \mathrm{mK}$. The DC voltage vs. current ( $V_{d c}$ vs. $I_{d c}$ ) characteristic of the junction at $T=30 \mathrm{mK}$ for a few different $V_{g}$ 's is also presented in Fig. 2b. As we increase $I_{d c}$ from zero, the junction is in its superconducting state and its resistance is zero. However, once $I_{d c}$ is increased above a critical value $\left(I_{C}\right.$, marked by an arrow in Fig 2b), the junction transitions from the superconducting state to a normal state with a non-zero resistance. The junction critical current, $I_{C}$, is highlighted by a white curve in Fig. 2a. First, we observe that $I_{C}$ is gate tunable, with larger $I_{C}$ for $V_{g}>V_{C N P}$. However, when $V_{g}$ is tuned near the charge neutrality point $\left(V_{C N P} \sim 4\right.$ V), $I_{C}$ decreases and eventually saturates for more negative $V_{g}$ 's as previously observed in $\mathrm{Bi}_{2} \mathrm{Se}_{3}$ flakes ${ }^{27}$ and graphene $e^{2829}$. One possible explanation for the saturation of $I_{C}$ for $V_{g}$ below the $V_{C N P}$ is that the $\mathrm{Nb}$ electrodes electron-dope the underlying material (TINR). Therefore, when $V_{g}<V_{C N P}$, a p-n junction is formed in the TINR. This p-n junction can weaken and eventually break the induced superconductivity as was shown in graphene ${ }^{30}$. Another plausible explanation may be the poor injection of the holes into TINR's by Nb, 


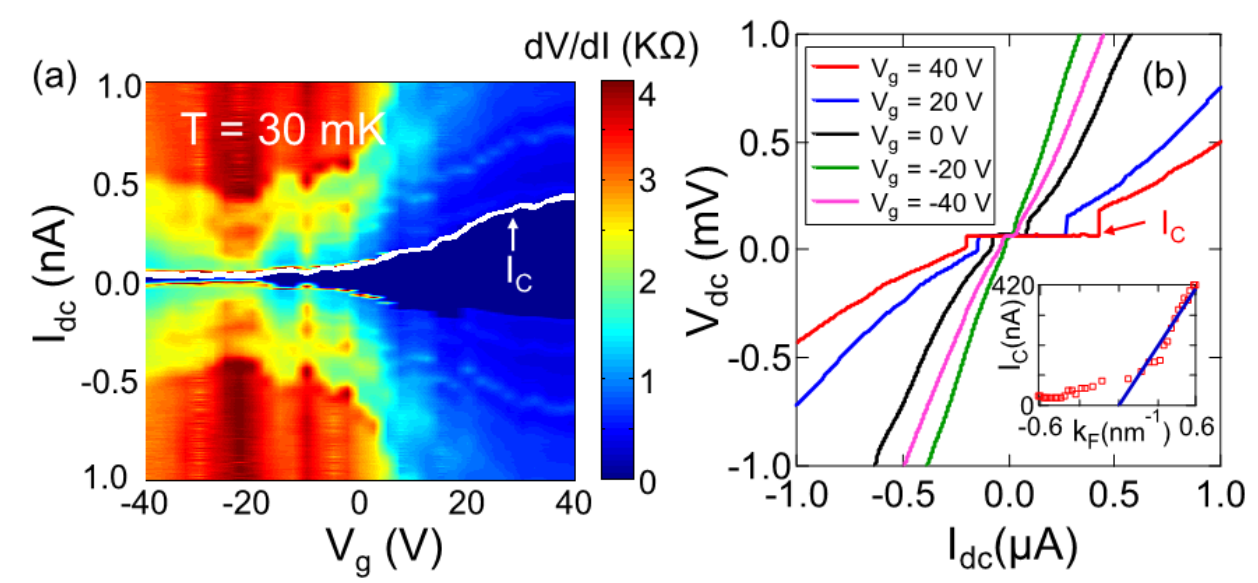

FIG. 2. (a) Color map of $d V / d I$ vs. $V_{g}$ and bias current $I_{d c}$ for $T=30 \mathrm{mK}$. Critical current $\left(I_{C}\right)$ is represented by a white trace on the colormap. (b) DC Voltage $\left(V_{d c}\right)$ vs. DC current $\left(I_{d c}\right)$ characteristic of the device for different $V_{g}$ 's at $T=30 \mathrm{mK}$. Inset: $I_{C}$ vs. $k_{F}$ (Fermi momentum). Blue curve is a linear fit for $k_{F}>0.4 \mathrm{~nm}^{-1}$. Data in (a) and (b) were measured with sweeping $I_{d c}$ from $-1 \mu A$ to $1 \mu A$.

as will be demonstrated from the low transparency of the contacts for $V_{g}<V_{C N P}$ from our analysis of MAR's (Fig. 3). The inset of Fig. 2b shows the dependence of $I_{C}$ on the Fermi momentum $\left(k_{F}\right)$, where $k_{F}=\sqrt{4 \pi C_{o x}\left(V_{g}-V_{C N P}\right) / e}$ and $C_{o x}$ is the parallel plate capacitance per unit area of a $300-\mathrm{nm} \mathrm{SiO} 2\left(\sim 12 \mathrm{nF} / \mathrm{cm}^{2}\right)$. For $k_{F}>0.4 \mathrm{~nm}^{-1}$, we observe $I_{C}$ varies linearly with $k_{F}$, as experimentally demonstrated in ballistic graphene Josephson junctions 31 . We also observe the junction critical temperature $\left(T_{C}\right.$, the temperature below which the junction resistance goes to zero and supercurrent starts to flow in the junction) changes with $V_{g}$ from $T_{C}=1.6 \mathrm{~K}$ for $V_{g}=40 \mathrm{~V}$ to $T_{C}=0.7 \mathrm{~K}$ for $V_{g}=10 \mathrm{~V}$. Using BCS theory, we extract the induced superconducting gap $(\Delta)$ in the TINR as $\Delta=1.76 k_{B} T_{C}=$ $242 \mu \mathrm{eV}$ and $106 \mu \mathrm{eV}$ for $V_{g}=40 \mathrm{~V}$ and $V_{g}=10 \mathrm{~V}$, respectively. We note that the resistance $(d V / d I)$ of the junction does not change as we increase $V_{d c}$ above $\Delta_{N b} / e(\sim 975 \mu \mathrm{V})$ and even slightly beyond $2 \Delta_{N b} / e$ as will be discussed later. As a result, the normal resistance $\left(R_{N}\right)$ in our junctions is obtained at $V_{d c}$ slightly above $\Delta_{N b} / e$. We obtain $I_{C} R_{N} \sim 304 \mu \mathrm{V}$ and $266 \mu \mathrm{V}$ for $V_{g}=40 \mathrm{~V}$ and $10 \mathrm{~V}$, respectively.

Fig. 3a displays $d I / d V$ vs. $V_{d c}$ for $V_{g}=40 \mathrm{~V}$ at $T=30 \mathrm{mK}$. Several peaks (within the $\mathrm{Nb}$ superconducting gap) in $d I / d V$ are observed at $V_{d c}=V_{n}=2 \Delta_{N b} /$ en (where $n=2,3,4,5,6,9$, and 13 ) as marked by the arrows in Fig. 3a. These $\mathrm{dI} / \mathrm{dV}$ peaks are consistent with MAR ${ }^{25}$. We note that these peaks are symmetric around $V_{d c}=0 \mathrm{~V}$ and thus below we focus only on the positive peaks. No feature in $\mathrm{dI} / \mathrm{dV}$ vs. $V_{d c}$ is identified for $n=1$ and $R_{N}$ is achieved for $V>\Delta_{N b} / e$ instead of $V>2 \Delta_{N b} / e$. The absence of the first $(n=1)$ MAR peak has been noted in some SNS junctions ${ }^{20126}$ and may be related to the presence of mid-gap zero-energy states as described elsewhere ${ }^{32 / 33}$. From the linear fit of $\mathrm{dI} / \mathrm{dV}$ peaks vs. $1 / n$, we obtain $\Delta_{N b} \sim 975 \mu \mathrm{eV}$, which is in excellent agreement with the $\Delta_{N b}$ obtained from the BCS theory and $T_{C}^{N b} \sim 6.5 \mathrm{~K}$. Moreover, the observed $\mathrm{dI} / \mathrm{dV}$ peaks are reproducible and independent of $V_{d c}$ sweep direction. While we do not observe any $\mathrm{dI} / \mathrm{dV}$ peaks corresponding to $n=7$ and 8 , higher-order peaks ( $n=9$ and 13) are present, a feature that needs further investigation. The observation of the high-order MAR peaks is an indication of high transparency of contacts in our junction.

Fig. $3 \mathrm{~b}$ depicts the differential conductance $\left(\mathrm{dI} / \mathrm{dV}\right.$, normalized by $\left.1 / R_{N}\right)$ vs. (positive) $V_{d c}$ for $\mathrm{T}=30 \mathrm{mK}$ at three different $V_{g}$ 's. First, we observe that the position of the $\mathrm{dI} / \mathrm{dV}$ peaks remains relatively constant with $V_{g}$, in contrast to the oscillatory behavior of $\mathrm{dI} / \mathrm{dV}$ peaks around a resonant level in a quantum dot $\mathrm{t}^{34 \mid 35}$. This suggests the absence of localized states in our TINR devices. The high-order dI/dV peaks observed for $V_{g}>V_{C N P}$ further indicate that the contacts are highly transparent. However, for $V_{g}<V_{C N P}$, the amplitude of the $\mathrm{dI} / \mathrm{dV}$ peaks decreases with more negative $V_{g}$, e.g. with vanishing peak amplitudes for $n=3,4,5,6$, and 9 at $V_{g}=-40 \mathrm{~V}$ (see Fig. 3c). It has been previously reported that 
(a)

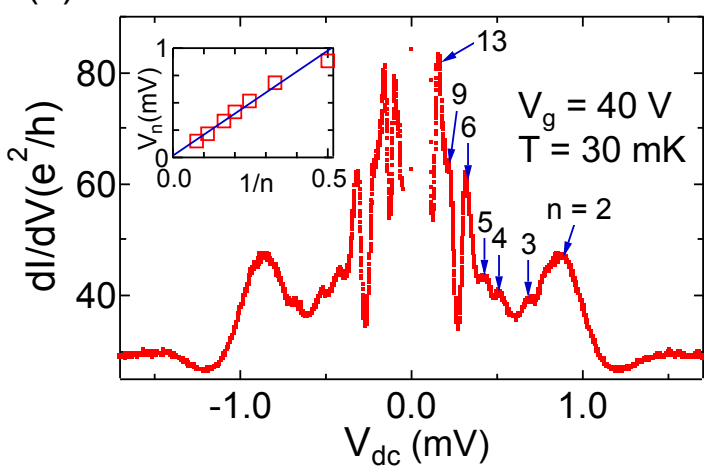

(b)

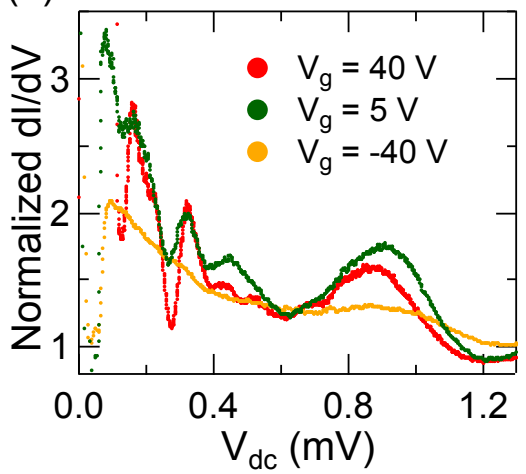

FIG. 3. (a) Differential conductance $(d I / d V)$ vs. $V_{d c}$ for $V_{g}=40 \mathrm{~V}$. Each $\mathrm{dI} / \mathrm{dV}$ peak position ( $V_{n}$, expected to be $2 \Delta_{N b} / e n$ ) is labeled with its index $n$, starting with $n=2$ for the peak near $V_{d c}=900 \mu \mathrm{eV}$. Inset: $V_{n}$ vs. $1 / n$. Solid line is a linear fit with a corresponding slope of $\sim 1.8$ meV, which agrees with the $2 \Delta_{N b}$ calculated from the BCS theory for the observed junction critical temperature $T_{C} \sim 6.5 K$. (b) $d I / d V$ normalized by $1 / R_{N}$ vs. $V_{d c}$ for three representative $V_{g}$ 's $=$ 40, -40 and $5 \mathrm{~V}$, corresponding to n-type, p-type and near the charge neutrality point. All the measurements were performed at $T=30 \mathrm{mK}$.

in JJS 36137 , the MAR peak amplitude depends on the ratio between $\xi$ (superconducting coherence length in the channel ${ }^{36}$ and L (channel length), with larger amplitudes for larger $\xi / \mathrm{L}$. For $V_{g}=40 \mathrm{~V}$, the amplitude of the $n=2$ peak in the normalized $\mathrm{dI} / \mathrm{dV}$ is $\sim 2$, indicating $\xi>\mathrm{L}$, which is also corroborated with the observation of supercurrent. Fig. 3c shows details of dI/dV vs. $V_{d c}$ curves (at positive side of $V_{d c}$ ) for three characteristic $V_{g}$ 's. The vanishing of $\mathrm{dI} / \mathrm{dV}$ peaks for $V_{g}<V_{C N P}$ may be related to the pinning of the Fermi level to the electron-doped regime under the $\mathrm{Nb}$ electrodes and hence the formation of p-n junctions for $V_{g}<V_{D P}$, as has been observed in graphene JJs 28129 .

Fig. 4a depicts the T-dependence of the $\mathrm{dI} / \mathrm{dV}$ (normalized by $1 / R_{N}$ ) vs. $V_{d c}$ for $V_{g}=$ $40 \mathrm{~V}$, exhibiting a reduction of the $\mathrm{Nb}$ superconducting gap with increasing T. Dashed lines are guides to the eyes corresponding to the expected T-dependence of $\mathrm{dI} / \mathrm{dV}$ peak positions $\left(V_{n}\right)$ from BCS theory. We observe a nearly flat and featureless $\mathrm{dI} / \mathrm{dV}$ vs. $V_{d c}$ for $\mathrm{T}=6.6$ $\mathrm{K}$ (slightly above $T_{C}^{N b} \sim 6.5 \mathrm{~K}$ ). We also observe that while $\mathrm{dI} / \mathrm{dV}$ peaks are noticeable up to high temperatures $\sim 5.2 \mathrm{~K}$ ), the amplitude of the peaks reduces with increasing $\mathrm{T}$, and some of the peaks merge together at higher $\mathrm{T}$ (e.g. peaks for $n=3$ and 4 merge at $\mathrm{T}=3.5 \mathrm{~K}$ ). Fig. $4 \mathrm{~b}$ shows the T-dependence of $V_{n}$ for $n=2,3,4$ and 6 . Using the BCS theory to fit $V_{n}$ vs. T, we extract a $T_{C} \sim 6 \mathrm{~K}$, in fair agreement with $T_{C}^{N b} \sim 6.5 \mathrm{~K}$. Fig. 4c displays the T-dependence of $\Delta_{N b}$ extracted from each $\mathrm{dI} / \mathrm{dV}$ peak (for $n=2,3,4$ and 6 ), where $\Delta_{N b}=n e V_{n}(T) / 2$, together with the fit of $\Delta_{N b}$ vs. T obtained from the BCS theory, which is seen to describe the data well.

We demonstrated Josephson junctions based on mechanically exfoliated bulk-insulating 3D topological insulator nanoribbons in proximity to superconducting $\mathrm{Nb}$ electrodes. We observe high-order $(n=13)$ multiple Andreev reflections, demonstrating charge transport in the TINR channel is coherent. Furthermore, the critical current exhibits gate effects and can be gate-tuned around one order of magnitude from $\sim 50 \mathrm{nA}$ to $\sim 430 \mathrm{nA}$ at $30 \mathrm{mK}$. Our measurements of supercurrent in Josephson junctions based on TINRs help to better understand the nature of induced superconductivity in these junctions and pave the way toward exploration of the envisioned topologically protected devices based on superconductorTINR-superconductor junctions.

\section{ACKNOWLEDGMENTS}

We acknowledge support from NSF (DMR \#1410942). The TI material synthesis was supported by DARPA MESO program (Grant N66001-11-1-4107). L.A.J. also acknowledges support by a Purdue Center for Topological Materials fellowship. L.P.R. and A.K. 

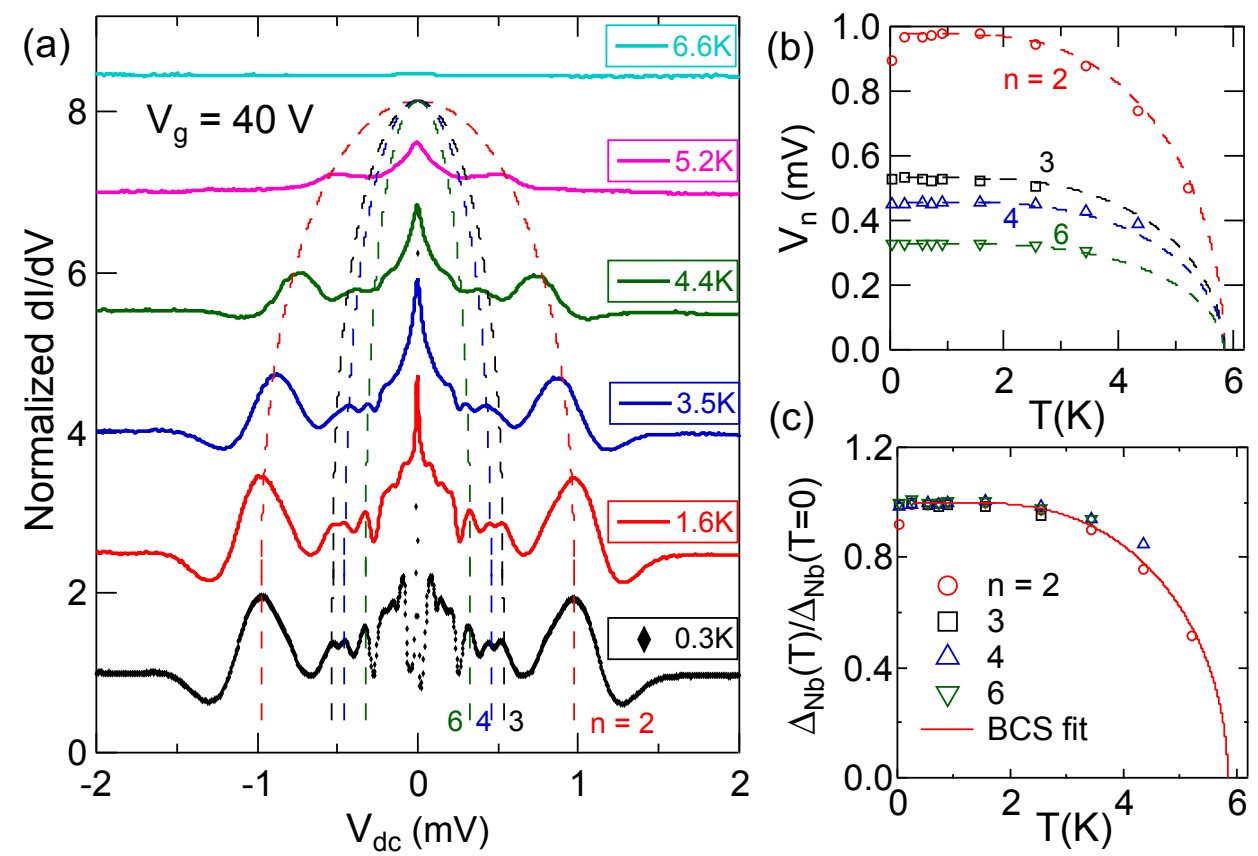

FIG. 4. (a) Normalized $d I / d V$ vs. $V_{d c}$ for different $T$ 's at $V_{g}=40 \mathrm{~V}$. Dashed lines are guides to the eyes corresponding to the expected $T$-dependence of $V_{n}$ from BCS theory for $n=2,3,4$ and 6. (b) $V_{n}$ vs. $T$ for $n=2,3,4$ and 6. Dashed lines are BCS fits. (c) Temperature dependence of normalized $\Delta_{N b} / \Delta_{N b}(T=0 K)$, where $\Delta_{N b}=e n V_{n}(T) / 2$ is obtained from different $d I / d V$ peaks corresponding to $n=2,3,4$ and 6 . Solid line is a BCS-theory fit.

acknowledge support from the U.S. Department of Energy under Award de-sc0008630.

${ }^{1}$ M. Z. Hasan and C. L. Kane, "Colloquium: Topological insulators," Reviews of Modern Physics 82 3045-3067 (2010)

${ }^{2}$ X.-L. Qi and S.-C. Zhang, "Topological insulators and superconductors," Reviews of Modern Physics 83, $1057-1110$ (2011)

${ }^{3} \mathrm{~L}$. Fu and C. L. Kane, "Superconducting proximity effect and Majorana fermions at the surface of a topological insulator," Physical Review Letters 100, 096407 (2008)

${ }^{4}$ A. Cook and M. Franz, "Majorana fermions in a topological-insulator nanowire proximity-coupled to an s-wave superconductor," Physical Review B 84, 201105 (2011).

${ }^{5}$ A. M. Cook, M. M. Vazifeh, and M. Franz, "Stability of Majorana fermions in proximity-coupled topological insulator nanowires," Physical Review B 86, 1-17 (2012)

${ }^{6}$ R. Ilan, J. H. Bardarson, H. S. Sim, and J. E. Moore, "Detecting perfect transmission in Josephson junctions on the surface of three dimensional topological insulators," New Journal of Physics 16, 053007 (2014).

${ }^{7}$ L. A. Jauregui, M. T. Pettes, L. P. Rokhinson, L. Shi, and Y. P. Chen, "Gate tunable relativistic mass and Berry's phase in topological insulator nanoribbon field effect devices," Scientific reports 5, 8452 (2015)

${ }^{8}$ L. A. Jauregui, M. T. Pettes, L. P. Rokhinson, L. Shi, and Y. P. Chen, "Magnetic field-induced helical mode and topological transitions in a topological insulator nanoribbon," Nature Nanotechnology 11, 345-351 (2016)

${ }^{9}$ B. Sacépé, J. B. Oostinga, J. Li, A. Ubaldini, N. J. Couto, E. Giannini, and A. F. Morpurgo, "Gate-tuned normal and superconducting transport at the surface of a topological insulator," Nature Communications 2, 575 (2011)

${ }^{10}$ F. Qu, F. Yang, J. Shen, Y. Ding, J. Chen, Z. Ji, G. Liu, J. Fan, X. Jing, C. Yang, and L. Lu, "Strong Superconducting Proximity Effect in $\mathrm{Pb}_{-} \mathrm{Bi}_{2} \mathrm{Te}_{3}$ Hybrid Structures," Scientific Reports 2, 339 (2012)

${ }^{11}$ M. Veldhorst, M. Snelder, M. Hoek, T. Gang, V. K. Guduru, X. L. Wang, U. Zeitler, W. G. van der Wiel, A. A. Golubov, H. Hilgenkamp, A. Brinkman, V. K. Guduru, U. Zeitler, W. G. v. d. Wiel, A. A. Golubov, H. Hilgenkamp, and A. Brinkman, "Josephson supercurrent through a topological insulator surface state," Nature materials 11, 417-421 (2012)

${ }^{12}$ J. R. Williams, A. J. Bestwick, P. Gallagher, S. S. Hong, Y. Cui, A. S. Bleich, J. G. Analytis, I. R. Fisher, and D. Goldhaber-Gordon, "Unconventional Josephson effect in hybrid superconductor-topological insulator devices," Physical Review Letters 109, 056803 (2012)

${ }^{13}$ I. Sochnikov, A. J. Bestwick, J. R. Williams, T. M. Lippman, I. R. Fisher, D. Goldhaber-Gordon, J. R. Kirtley, and K. A. Moler, "Direct measurement of current-phase relations in superconductor/ topological insulator/ superconductor junctions." Nano letters 13, 30863092 (2013)

${ }^{14}$ J. B. Oostinga, L. Maier, P. Schüffelgen, D. Knott, C. Ames, C. Brüne, G. Tkachov, H. Buhmann, and 
L. W. Molenkamp, "Josephson supercurrent through the topological surface states of strained bulk HgTe," Physical Review X 3, 021007 (2013)

${ }^{15}$ A. D. K. Finck, C. Kurter, Y. S. Hor, and D. J. V. Harlingen, "Phase Coherence and Andreev Reflection in Topological Insulator Devices," Physical Review X 4, 041022 (2014).

${ }^{16}$ J. H. Lee, G.-H. Lee, J. Park, J. Lee, S.-G. Nam, Y.-S. Shin, J. S. Kim, and H.-J. Lee, "Local and nonlocal Fraunhofer-like pattern from an edge-stepped topological surface josephson current distribution," Nano letters 14, 5029-5034 (2014)

${ }^{17}$ L. Yang, X. Cui, J. Zhang, K. Wang, M. Shen, S. Zeng, S. a. Dayeh, L. Feng, and B. Xiang, "Lattice strain effects on the optical properties of MoS2 nanosheets." Scientific reports 4, 5649 (2014)

${ }^{18}$ D. Zhang, J. Wang, A. M. Dasilva, J. S. Lee, H. R. Gutierrez, M. H. W. Chan, J. Jain, and N. Samarth, "Superconducting proximity effect and possible evidence for Pearl vortices in a candidate topological insulator," Physical Review B 84, 165129 (2011)

${ }^{19}$ C. Kurter, a. D. K. Finck, Y. S. Hor, and D. J. Van Harlingen, "Evidence for an anomalous current-phase relation in topological insulator Josephson junctions," Nature Communications 6, 7130 (2015)

${ }^{20}$ J. Wiedenmann, E. Bocquillon, R. S. Deacon, S. Hartinger, O. Herrmann, T. M. Klapwijk, L. Maier, C. Ames, C. Brüne, C. Gould, A. Oiwa, K. Ishibashi, S. Tarucha, H. Buhmann, and L. W. Molenkamp, " $4 \pi$-periodic Josephson supercurrent in HgTe-based topological Josephson junctions," Nature communications 7, $10303(2016)$

${ }^{21}$ M. P. Stehno, V. Orlyanchik, C. D. Nugroho, P. Ghaemi, M. Brahlek, N. Koirala, S. Oh, and D. J. V. Harlingen, "Signature of a topological phase transition in the Josephson supercurrent through a topological insulator," Physical Review B 93, 035307 (2016)

${ }^{22}$ R. S. Deacon, J. Wiedenmann, E. Bocquillon, F. Domínguez, T. M. Klapwijk, P. Leubner, C. Brüne, E. M. Hankiewicz, S. Tarucha, K. Ishibashi, H. Buhmann, and L. W. Molenkamp, "Josephson Radiation from Gapless Andreev Bound States in HgTe-Based Topological Junctions," Physical Review X 7, 021011 (2017)

${ }^{23}$ Y. Xu, I. Miotkowski, C. Liu, J. Tian, H. Nam, N. Alidoust, J. Hu, C.-K. Shih, M. Z. Hasan, and Y. P. Chen, "Observation of topological surface state quantum Hall effect in an intrinsic three-dimensional topological insulator," Nature Physics 10, 956-963 (2014)

${ }^{24}$ Y. Xu, I. Miotkowski, and Y. P. Chen, "Quantum transport of two-species Dirac fermions in dual-gated three-dimensional topological insulators," Nat Commun 7, 11434 (2016)

${ }^{25} \mathrm{M}$. Tinkham, Introduction to superconductivity (Dover Publications, 2004) p. 454.

${ }^{26}$ J. Xiang, A. Vidan, M. Tinkham, R. M. Westervelt, and C. M. Lieber, "Ge/Si nanowire mesoscopic Josephson junctions," Nature Nanotechnology 1, 208-213 (2006)

${ }^{27}$ S. Cho, B. Dellabetta, A. Yang, J. Schneeloch, Z. Xu, T. Valla, G. Gu, M. J. Gilbert, and N. Mason, "Symmetry protected Josephson supercurrents in three-dimensional topological insulators," Nature Communications 4, 1689 (2013)

${ }^{28}$ M. Ben Shalom, M. J. Zhu, V. I. Fal'ko, A. Mishchenko, A. V. Kretinin, K. S. Novoselov, C. R. Woods, K. Watanabe, T. Taniguchi, A. K. Geim, and J. R. Prance, "Quantum oscillations of the critical current and high-field superconducting proximity in ballistic graphene," Nature Physics 12, 318-322 (2015)

${ }^{29}$ V. E. Calado, S. Goswami, G. Nanda, M. Diez, A. R. Akhmerov, K. Watanabe, T. Taniguchi, T. M. Klapwijk, and L. M. K. Vandersypen, "Ballistic Josephson junctions in edge-contacted graphene," Nature Nanotechnology 10, 761-765 (2015)

${ }^{30}$ J.-H. Choi, G.-H. Lee, S. Park, D. Jeong, J.-O. Lee, H.-S. Sim, Y.-J. Doh, and H.-J. Lee, "Complete gate control of supercurrent in graphene pn junctions," Nature Communications 4, 1-10 (2013)

${ }^{31}$ N. Mizuno, B. Nielsen, and X. Du, "Ballistic-like supercurrent in suspended graphene Josephson weak links," Nature Communications 4, 2716 (2013)

${ }^{32}$ D. M. Badiane, M. Houzet, and J. S. Meyer, "Nonequilibrium Josephson effect through helical edge states," Physical Review Letters 107, 177002 (2011).

${ }^{33}$ P. San-Jose, J. Cayao, E. Prada, and R. Aguado, "Multiple Andreev reflection and critical current in topological superconducting nanowire junctions," New Journal of Physics 15, 075019 (2013)

${ }^{34}$ M. R. Buitelaar, W. Belzig, T. Nussbaumer, B. Babić, C. Bruder, and C. Schönenberger, "Multiple Andreev Reflections in a Carbon Nanotube Quantum Dot," Physical Review Letters 91, 057005 (2003)

${ }^{35}$ P. Jarillo-Herrero, J. A. van Dam, and L. P. Kouwenhoven, "Quantum supercurrent transistors in carbon nanotubes," Nature 439, 953-956 (2006)

${ }^{36}$ X. Du, I. Skachko, and E. Y. Andrei, "Josephson current and multiple Andreev reflections in graphene SNS junctions," Physical Review B 77, 184507 (2008).

${ }^{37}$ M. Kjærgaard, H. J. Suominen, M. Nowak, A. Akhmerov, J. Shabani, C. Palmstrøm, F. Nichele, and C. M. Marcus, "Transparent semiconductor-superconductor interface and induced gap in an epitaxial heterostructure josephson junction," Physical Review Applied 7, 034029 (2017). 